\title{
Research on the Design and Implementation of Smart Home System Based on ARM and ZigBee Techniques with the Analysis of Data Transmission Security Enhancement Model
}

\author{
Tuanbu Wang ${ }^{1, a}$ and Wenzhun Huang ${ }^{1, b}$ \\ ${ }^{1}$ Xijing University, Xi'an, Shaanxi, China \\ a597669319@qq.com, ${ }^{b} 1051917312 @ q q . c o m$
}

Keywords: Smart home system; ARM and ZigBee; Data transmission; Security enhancement

\begin{abstract}
In this paper, we combine the technique of ARM and ZigBee to design and implement the novel smart home system. Through analyzing the data transmission security enhancement model, we modify the traditional model with security concern. Each smart home system can be controlled by the server to facilitate access to residential property services, municipal services, such as weather forecast information. While using ZigBee as household interior various network can be easily will not have communication interface devices connected to the Internet gateway, does not need complex wiring or buy expensive appliances with communication interface. In the future research, we will numerically experiment our system and collect feedback for further optimization.
\end{abstract}

\section{Introduction}

Smart home is a house played as a platform with the general use of integrated wiring technology, communication technology and automatic control technology and related technical implementation household facilities connected. Building intelligent residential facility management system so as to realize safe and comfortable living environment and environmental protection and the energy saving. Information community and home intelligent network will become the future trend of the housing construction in our country. Due to the complexity of cable wiring, the line is more hidden trouble and integrated, intelligent degrees is not high, thus wireless blue-tooth communication is the focus of the study by communications industry in recent years. Bluetooth module in communication, electronics, home wireless network and has more applications in such aspects as personal information network. With the further development of the wireless blue-tooth technology and the continuous expansion of products, technology matures and prices decline, blue-tooth technology is gradually applied to the industrial field and in the family. Because wireless blue-tooth communication medium particularity and advantage, it is a good way to flexibly applied to smart home information network [1].

However, the most essential challenge for the blue-tooth communication is transmission security. The emergence of the concept of Internet of things provides the new direction for the development of smart home. Through the installation of various sensors to collect within the residential environment, equipment and the personnel information, using ZigBee wireless network to the above all kinds of information access Internet gateway again by the gateway to forward this information to the Internet in the server. The user could through the browser or client software on the mobile phone or computer login server can real-time information of each subsystem and control the operation of the household equipment so as to construct a smart home system based on Internet of things [2].

In the modern design pattern of the smart home, the primary orientations and steps could be listed as the follows. (1) Records in the database and the users belong to the user's detailed information and the two users can mutual interference between electrical equipment, dynamic addition. When we get in the database when a user task. The task is passed down through the communication interface. The advantage is that the server communication program can only write once and provides users and user family internal control two parts used with simplifies the system structure of communication. (2) Customer query control terminal mainly through a browser should also be a mobile phone, PDA and other terminal equipment, control commands and backend server through wired and wireless network 
communication. Backend server to verify the identity of the user and task into the database query control records and parameters. (3) Within the family network system because of the need to control the hardware, so they need the complete system resident program control switch such as state signal discrete devices. In the Fig. 1, we demonstrate the organization of the modern smart home.

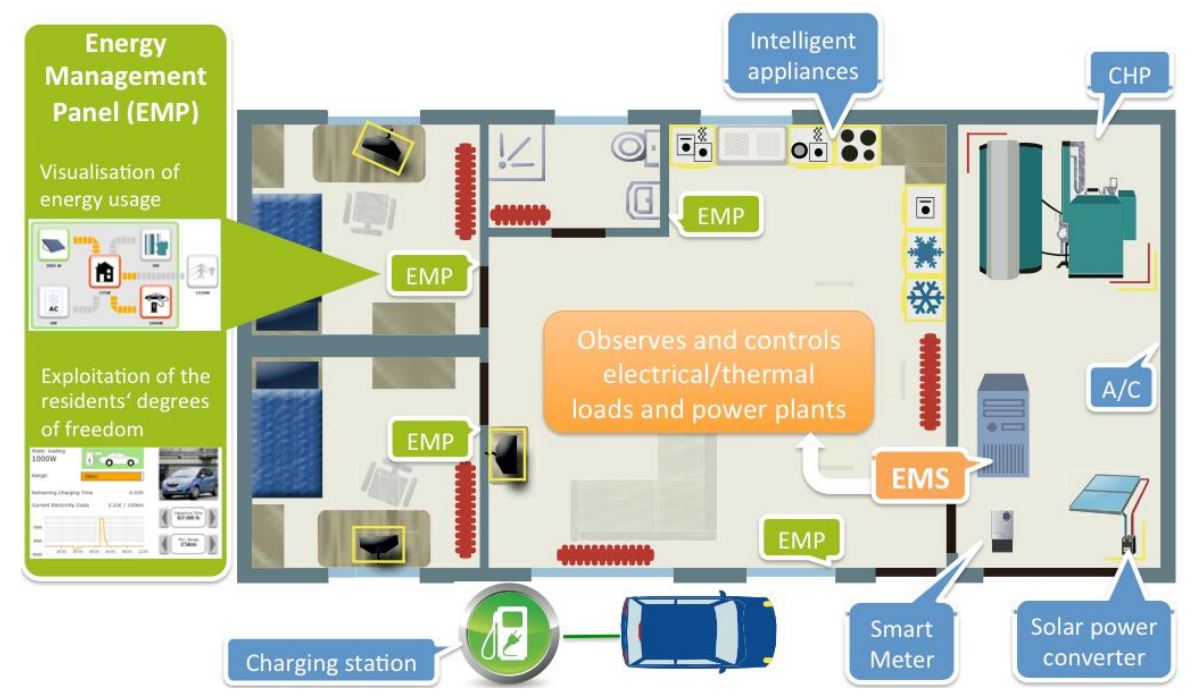

Figure 1. The topology and structure of the modern smart home system

In this paper, we conduct research on the design and implementation of smart home system based on ARM and ZigBee techniques with the analysis of data transmission security enhancement model. The rest of the paper is organized as the follows. (1) In the part one, we demonstrate basic technique of ZigBee and applications. (2) In the part two, we introduce the ARM technique with the general use of the smart system. (3) We model the data transmission algorithm and optimize with the enhanced security model. In the final part, we simulate the system with the final discussion.

\section{The Core Techniques of the Proposed Method}

The ZigBee Technique for the Smart Home. Intelligent gateway is the core part of the smart home system, its main function is as the Internet and the Internet and family ZigBee wireless network data exchange station of all intelligent home appliance in the home node equipment data are sent to the gateway and the intelligent gateway protocol conversion through the Internet after sent to the mobile terminal. Mobile terminal sends data through the Internet protocol conversion should be carried out by the gateway and also sent to the ZigBee network household appliance again. The advantages and the characteristics of the ZigBee technique could be summarized as the follows. (1) Low cost and the royalties from the agreement, and each chip price is about $\$ 2$ along with the progress of the process and technology, the cost will be further reduced. Low power consumption in the low-power standby mode, two ordinary dry-cell batteries can support the node work six months to two years longer, we can say quite natural conditions to devolve battery and the ordinary speed. (2) Short time delay, its response speed faster, general $15 \mathrm{~ms}$ from the sleep into working state. High capacity, the technology can be used, star, tree, and the mesh network structure is managed by a master node number of child nodes, up to a master node can manage 254 child nodes and at the same time, the master node can also be composed of a layer of network node management. (3) High security, it provides the three security pattern including no security setting, use the access control list to prevent illegal access to data, and use advanced encryption standard symmetrical password, flexible to determine its security properties.

Home gateway is the core of smart home and abstracts it as the family and family net network communication channel and is responsible for the basic communication protocol conversion between different devices and it also as service platform to provide users with a variety of possible value-added 
services. ZigBee module of software is divided into ZigBee coordinator program and ZigBee terminal nodes. The smart grid close integration of which is ZigBee network coordinator, ZigBee coordinator main work is responsible for the establishing, maintaining and management network with distribution network address, so the intelligent gateway ZigBee application process is more complex. All kinds of household appliances ZigBee network terminal nodes in order to realize the main functions are to join the intelligent gateway to establish ZigBee LAN, sensor data and intelligent gateway communication to upload sensor data to the intelligent gateway and receive intelligent gateway distributed execution control instruction. Intelligent gateway ZigBee node is the manager of the household ZigBee network. In the Fig.2, we demonstrate the general organization and node distribution of the ZigBee network.

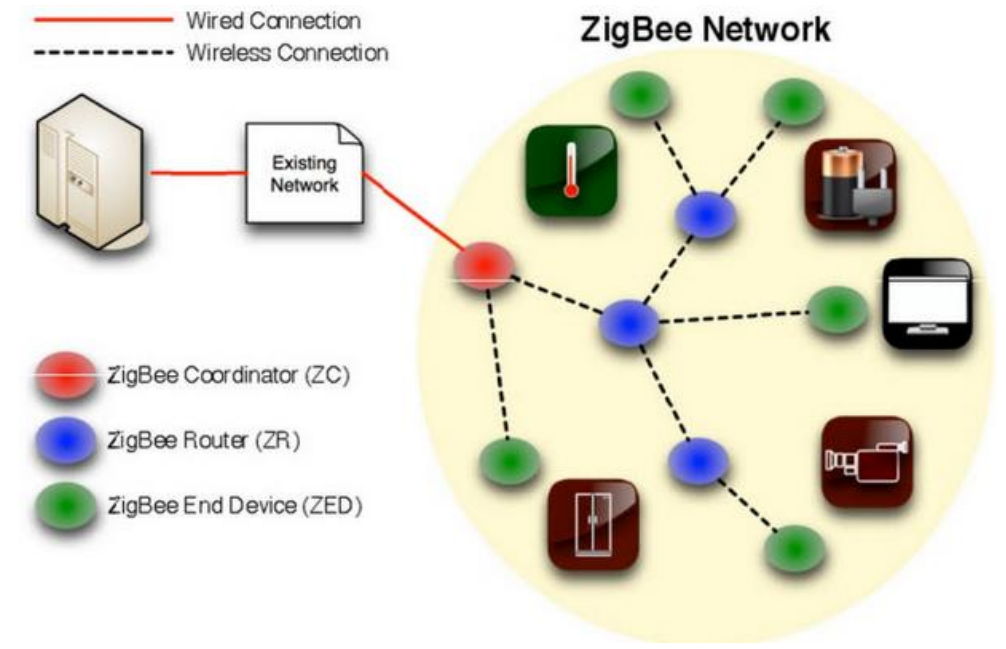

Figure 2. The general organization and node distribution of the ZigBee network

The ARM for the Smart Home System. Compared with ordinary household, smart home not only has the traditional residential function, provide safe, high grade and comfortable delightful family living space. It will also be the passive static structure into a dynamic intelligent tool and provides the directional information exchange function to help keep families and external information exchange and optimize people's life style, help people to arrange time effectively to enhance security of home life, even for a variety of energy cost savings. After the normal work of system initialization, timing acquisition sensor data, if it is abnormal, we collect data to control the interior of the corresponding equipment to the specified index and then sends data from selective through GSM or alarm achieve the goal of effective control of indoor equipment that also can control room equipment operation by the way of SMS. In the Fig. 3, we demonstrate the sample smart home control system base board.

Intelligent home control system generally consists of the sensor nodes, the coordinator node and embedded gateway, mobile phone or smart mobile terminals made up of several parts. From the network level is divided into the remote control and home control parts, respectively corresponding to the Internet and ZigBee wireless communication network. The embedded gateway is the core part of smart home control system mainly to complete the control part of information exchange and the core information sharing between sensor nodes and Internet exchange between wireless communication networks. Through the GPRS combined with wireless Internet, users can through phone or the remote login to the embedded gateway, intelligent mobile terminal to access system on sensor node in the system for data access or control. In sum, our system could be summarized as the follows [3-4].

$>$ Input module. Includes two kinds, one is measuring the parameter acquisition module, the fire temperature acquisition module, security camera monitoring module, acquisition and wireless transmission monitoring parameters. Control modules, namely the Internet or mobile phone user information was realized through network monitoring system and monitoring parameters setting, monitoring parameters in query, display and fortification such as instruction issued. 
Processing module. ARM development board as the core of master control board received by the data from the input module and logical judgment. If there is an exception occurs, inform the output module to make corresponding processing at the same time we also can according to the web page was sent on a variety of the settings and control instruction and then call the corresponding processing functions [5].

$>$ Output module. Analytical signal from the processing module and corresponding exception handling measures. If the temperature sensors measured temperature higher than the pre-set temperature or infrared sensors to detect criminals broke into, then open the corresponding sound and light alarm and corresponding exception handling and by controlling the camera to capture images and image processing with the data stored in the SQLite database.

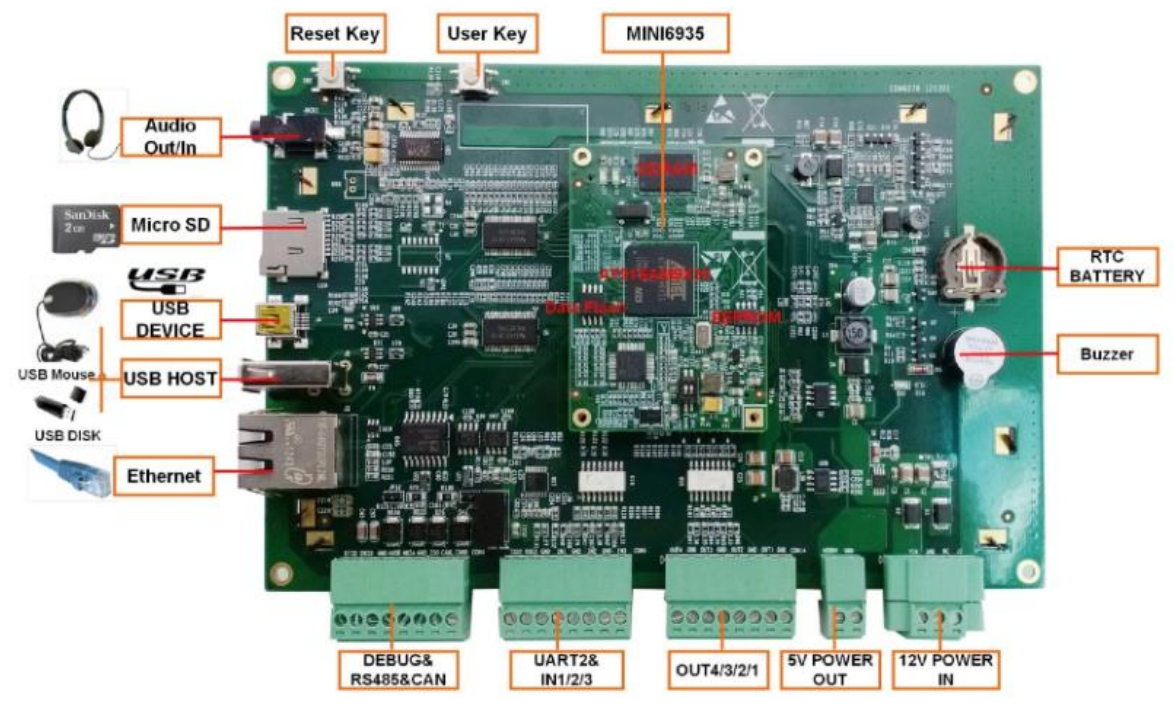

Figure 3. The sample smart home control system base board

The Data Transmission Security Enhancement Model. With the rapid development of network applications including the security problems have also been involved more and more attention, almost all the recent development of network applications are embedded into the corresponding security technology integration or measures, the application system to fully ensure confidentiality, integrity and availability of resources. The data transmission safety challenges could be summarized as the follows. (1) Denial of service, in the physical or the agreement on interference user data and signaling data and control data in the wireless transmission link on the right or to send a lot of illegal information server resources to make the legal users can't normal communication and realizes denial of service attacks. (2) Camouflage, disguised as a network unit captures user data and signaling data and control data with the pseudo terminal cheat network access services. Hacking or service network eavesdropping user data in the wireless link, signaling data and control data. (3) Deny the user denied that business expenses, business data sources and other users to send or receive data, network unit deny network services. Unauthorized access service, abuse of authority for access to unauthorized services. (4) Traffic analysis, active or passive traffic analysis so as to obtain the information time, rate, length, source and destination to become harmful [6].

Integrity is an information security requirement. It's different with confidentiality, the privacy is to prevent information leaked to unauthorized people and integrity requirements information and order are not destroyed and modify the content of the recipients can tell when or information change. If the lack of the integrity of the information and messages from the sender cannot guarantee its match the recipient receives the message. Integrity, general information can be extracted through the way to get news. SMS gateway must meet the requirements of the user communication at any time, this requires the SMS gateway has better robustness and higher ability to process and forward data. Due to short message transmission is in the network environment, SMS gateway received message may come from 
legitimate users that also may come from illegal users. SMS gateway need to take the appropriate measures will be in front of them apart. This means that when the illegal users use this means to pretend to be legitimate users to enjoy free services or charge from the user will be SMS gateway timely discover and refused to provide services.

To enhance the data transmission security, we adopt the popular message-digest algorithm to deal with the issues. Algorithm can obtain a random length of information to produce a 128 bit information summary, the information referred to as the hash value. Hash value is a unique and extremely compact numerical representation. If the hash and even change the paragraph only one letter, then the hash will produce different values. To find the hash to the same value of two different inputs, the calculation is almost impossible, so the hash value of the data can verify the integrity of the data. In this system requires a lot of key exchange, the calculation of key exchange can be used in a different way, such as the different content of message encryption, message authentication code and the hash function [7-8].

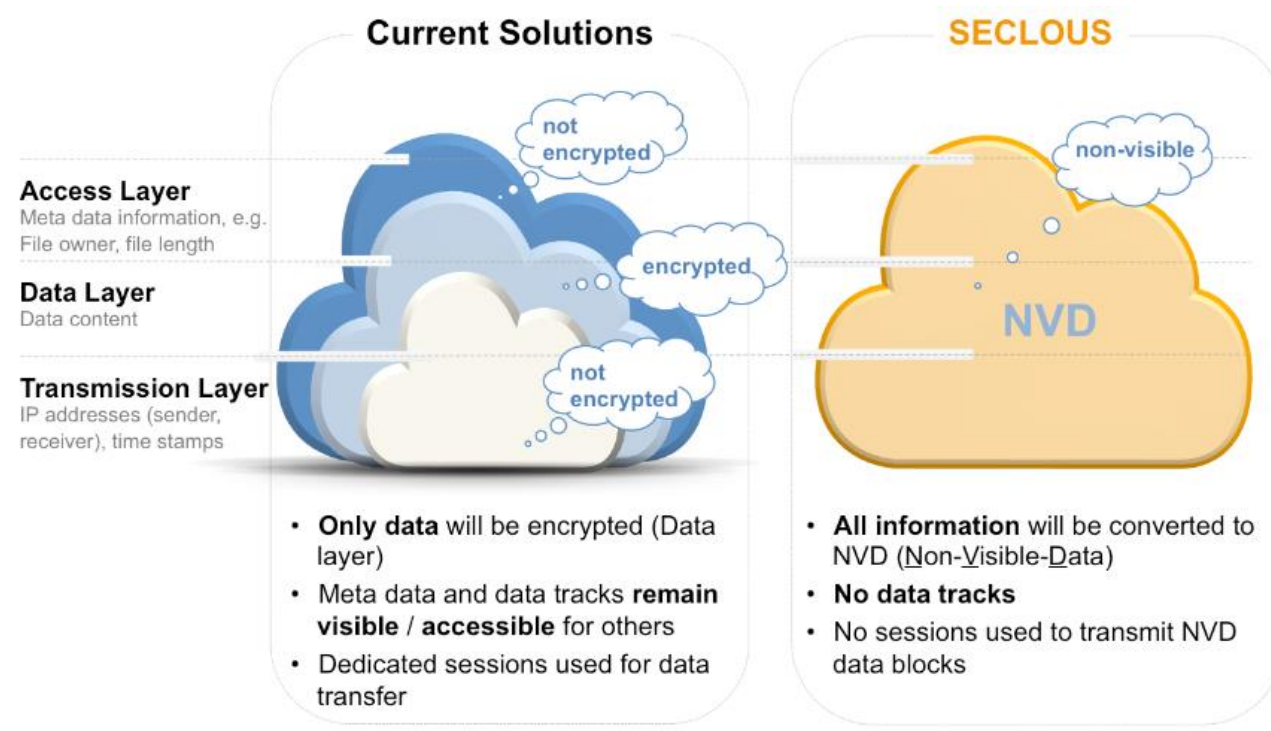

Figure 4. The data transmission security enhancement model

\section{Summary}

This article introduces design and implementation of smart home system based on ARM and ZigBee techniques with the analysis of data transmission security enhancement model. In the development of computer technology and intelligent wireless demand under the increasing of long operation, the traditional manual management and cable control technology is difficult to meet the requirements of convenience and efficiency of operation, therefore, the current household management of users in the intelligent monitoring technology and the wireless control system design. Our research provides the industry with novel solution for the system design and implementation.

\section{Acknowledgments}

This work is supported by the scientific research fund of Xijing University (No. XJ140234).

\section{References}

[1] Jiang, Hua, Jianhui Lin, and Changdong Wu. "Design of Smart Home Monitoring System Based on ARM and Power Line Carrier Communication." safety 10.7 (2015). (In Chinese)

[2] Zhao, Jianhua, and Zhantao ZHANG. "Portable Smart Home Terminal System Based on ARM and ZigBee." Computer \& Digital Engineering 9 (2013): 038. (In Chinese) 
[3] Wang, Jian Min, and Hai Bo Wei. "Design of smart home management system based on GSM and Zigbee." In Advanced Materials Research, vol. 842, pp. 703-707. 2014. (In Chinese)

[4] Ying, Yiming, and Peng Li. "Distance metric learning with eigenvalue optimization." The Journal of Machine Learning Research 13, no. 1 (2012): 1-26. (In Chinese)

[5] Wang, Haoxiang, Ferdinand Shkjezi, and Ela Hoxha. "Distance metric learning for multi-camera people matching." In Advanced Computational Intelligence (ICACI), 2013 Sixth International Conference on, pp. 140-143. IEEE, 2013. (In Chinese)

[6] Yu, Jun, Dapeng Tao, Jonathan Li, and Jun Cheng. "Semantic preserving distance metric learning and applications." Information Sciences 281 (2014): 674-686. (In Chinese)

[7] Hu, Youfan, Yan Zhang, Chen Xu, Long Lin, Robert L. Snyder, and Zhong Lin Wang. "Self-powered system with wireless data transmission." Nano Letters 11, no. 6 (2011): 2572-2577. (In Chinese)

[8] Kiani, Mehdi, and Maysam Ghovanloo. "Pulse delay modulation (PDM) a new wideband data transmission method to implantable medical devices in presence of a power link." In Biomedical Circuits and Systems Conference (BioCAS), 2012 IEEE, pp. 256-259. IEEE, 2012.

[9] Zhang Chengfa, Yang Feng. "The design and implementation of smart home remote monitoring system."Shanxi Electronic Technology,no. 6(2010),pp.45-47.(In Chinese)

[10]Zhan Baorong, Yu Xichang."The design of the wireless smart home network system."Wireless communication technology.no. 6(2010),pp.92-95.(In Chinese) 